validate the DVI approach as reflective of natural infection. The datasets provided here serve as a valuable resource for secondary analyses and that could be included in integrated meta-analyses of multicohort studies employing similar methodologies. This study and future systems analyses will help to elucidate the complex host response to this debilitating parasitic infection.

Prasida Holla ${ }^{1}$ and Tuan M. Tran (D) 2,3凶 ${ }^{1}$ Laboratory of Immunogenetics, National Institute of Allergy and Infectious Diseases, National Institutes of Health, Rockville, MD, USA. ${ }^{2}$ Division of Infectious Diseases, Department of
Medicine, Indiana University School of Medicine, Indianapolis, IN, USA. ${ }^{3}$ Ryan White Center for Pediatric Infectious Diseases and Global Health, Department of Pediatrics, Indiana University School of Medicine, Indianapolis, IN, USA.

$凶_{e-m a i l: t u a n t r a n @ i u . e d u}$

Published online: 22 April 2021

https://doi.org/10.1038/s41590-021-00917-1

\section{References}

1. Moncunill, G. et al. Sci. Transl. Med. 12, eaay8924 (2020).

2. Tran, T. M. et al. Immunity 51, 750-765.e10 (2019).

3. de Jong, S. E. et al. Nat. Immunol. https://doi.org/10.1038/s41590021-00911-7 (2021)

4. Crompton, P. D. et al. Annu. Rev. Immunol. 32, 157-187 (2014).

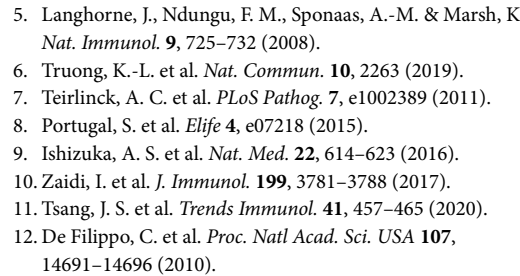

Acknowledgements

The authors would like to thank Austin J. Athman (Visual Medical Arts, National Institute of Allergy and Infectious Diseases, National Institutes of Health) for assistance with creating the illustration in Fig. 1.

Competing interests

The authors declare no competing interests.

\title{
Immunity after SARS-CoV-2 infections
}

\author{
T cell responses probably play important roles in the control of SARS-CoV-2 infection, but they have been relatively \\ understudied. Data now suggest that the majority of infected individuals develop robust and long-lasting $T$ cell \\ immunity, which has implications for the durability of immunity and future vaccine approaches.
}

\section{Prasanna Jagannathan and Taia T. Wang}

S ARS-CoV-2 infections result in highly heterogeneous clinical outcomes, ranging from the absence of any symptoms to severe disease and death. Given the fitness of this virus in humans thus far and its likely persistence in this reservoir, it is important to understand the quality and durability of immune memory that is elicited by infection. Although several studies have reported that individuals develop robust SARS-CoV-2-specific memory T cell responses following natural infection ${ }^{1-5}$, whether these responses correlate with clinical or immunologic outcomes has remained unclear. In the current issue of Nature Immunology, Zuo et al. ${ }^{6}$ characterize the durability and diversity of memory $T$ cell responses established after asymptomatic or mild COVID-19.

The authors studied $\mathrm{T}$ cell responses six months after infection in 100 individuals (median age 41 years) who had relatively mild infections (56 people) or asymptomatic infections (44 people). To enumerate $T$ cells that recognized SARS-CoV-2, cells from previously infected individuals were first stimulated with peptides from SARS-CoV-2 proteins to elicit a cytokine response. SARS-CoV2 -reactive T cells were then counted on the basis of secretion of the proinflammatory cytokine interferon (IFN)- $\gamma$ in an ELISpot (enzyme-linked immune absorbent spot) assay. Nearly all donors had a SARS-CoV2 -reactive $\mathrm{T}$ cell response in this assay. However, the magnitude of the responses was highly variable within the cohort, and a correlate of the response was the presence of symptoms early in the infection. People with symptomatic SARS-CoV-2 infections had IFN- $\gamma$-producing $\mathrm{T}$ cell responses of significantly higher magnitude at six months post-infection as compared to those with asymptomatic infection. Although individuals with severe disease were not included in this study, another recent report did not find significant differences in the magnitude of SARS-CoV2 -specific $T$ cell responses between participants who were hospitalized and those who were not hospitalized ${ }^{5}$. Importantly, IFN- $\boldsymbol{\gamma}$-producing T cell responses did not correlate with the age of the subjects within the cohort. Together with a separate report that found that $\mathrm{T}$ cell responses in patients with COVID-19 increased with age ${ }^{7}$, the data are reassuring that robust $\mathrm{T}$ cell responses can be elicited by SARS-CoV-2 infection regardless of one's age.
Next, the quality of the T cell response was characterized in greater granularity by quantifying virus-specific $\mathrm{CD}^{+}$and $\mathrm{CD}^{+} \mathrm{T}$ cell subsets and the production of intracellular cytokines (IFN- $\gamma$, interleukin (IL)-2, IL-4 and tumor necrosis factor) by these cells. SARS-CoV-2-specific $\mathrm{CD}^{+} \mathrm{T}$ cells were approximately twice as abundant as $\mathrm{CD}^{+} \mathrm{T}$ cells, and cytokine production differed between these populations; for example, more IL-2 was produced in $\mathrm{CD}^{+} \mathrm{T}$ cells, but more IFN- $\gamma$ was produced in $\mathrm{CD}^{+} \mathrm{T}$ cells. The greater $\mathrm{CD}^{+}$versus $\mathrm{CD}^{+} \mathrm{T}$ cell response is consistent with findings from a separate report ${ }^{5}$, which analyzed 43 patients $6-8$ months after infection, and is independent of age, sex or whether the initial infection was symptomatic. Interestingly, IL-2, with or without IFN- $\gamma$, was the dominant $\mathrm{CD} 4^{+}$ cytokine produced in response to both spike (S) and non-spike protein stimulation, and this was confirmed through analysis of ELISpot cell culture supernatants.

Zuo et al. next studied whether the magnitude of the T cell IFN- $\gamma$ response at six months after infection correlated with the antibody response over time. A nice feature of this study was the monthly characterization of antibodies 


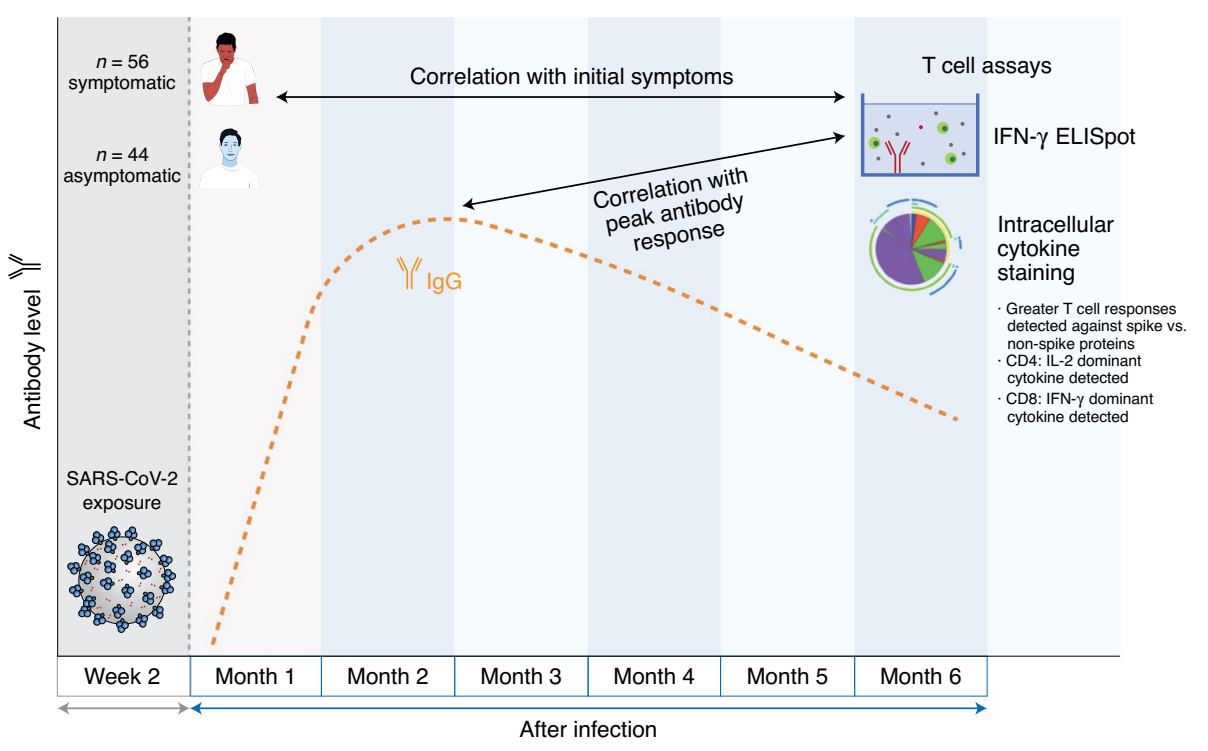

Fig. 1 | Correlations between SARS-CoV-2-specific T cell responses, disease severity and peak antibody response. Zuo et al. measured SARS-CoV-2-specific T cell responses in 100 individuals six months after infection. SARS-CoV-2-specific T cell responses were measured by IFN- $\gamma$ ELISpot and intracellular cytokine staining and correlated with both initial symptoms and the peak antibody response.

after diagnosis. This enabled a kinetic analysis of the levels of IgG specific for the SARS-CoV-2 S protein and nucleoprotein (N). These levels were highly heterogeneous among study participants. On average, IgG responses began to wane after approximately two months but remained well above the limit of detection in most subjects at month five. Interestingly, a greater magnitude of the S-specific T cell response at six months correlated with higher peak antibody levels against the $\mathrm{S}$ and $\mathrm{N}$ proteins and a sustained antibody response against the $\mathrm{N}$ protein. It remains to be determined whether the $\mathrm{T}$ cell response is associated with other antibody characteristics (for example, neutralizing capability and/or other effector functions) and/or whether other features of the $\mathrm{T}$ cell response (for example, $\mathrm{CD} 4^{+} \mathrm{T}$ cell production of IL-2) are also associated with the peak antibody response.

While the $\mathrm{T}$ cell response was assessed in this study six months after the infection, it is unclear whether any participants had been reinfected during that time. This is unlikely, given the low rate of reinfections within six months of a primary SARS-CoV-2 infection, but this could have influenced measurements in some participants ${ }^{8}$. In addition, although the authors assessed the $\mathrm{T}$ cell response in relation to the earlier antibody response, it will be important for future studies to assess whether the early $\mathrm{CD}^{+} \mathrm{T}$ cell response predicts the quality and/or durability of the antibody response over time. For example, T follicular helper $\left(\mathrm{T}_{\mathrm{FH}}\right)$ cells are thought to play a critical role in providing $B$ cell help and in the formation of humoral immune memory, and circulating populations of these cells that correlate with the development of neutralizing antiviral antibodies have been identified ${ }^{9}$. A recent paper demonstrated that the frequencies of circulating SARS-CoV-2-specific CD40L ${ }^{+} \mathrm{OX} 40^{+} \mathrm{T}_{\mathrm{FH}}$ cells were stable over several months ${ }^{5}$. It will be important to know whether the magnitude or quality of antigen-specific circulating $\mathrm{T}_{\mathrm{FH}}$ cells or other cell types at early time points predicts subsequent antibody quality and/or durability.

That the majority of $\mathrm{CD}^{+}$and $\mathrm{CD}^{+}$ $\mathrm{T}$ cells were activated by non-S viral epitopes has implications for vaccination in populations that have experienced prior SARS-CoV-2 infections. To engage these non-S-reactive $T$ cells that were established during infections, proteins besides $S$ could be incorporated into vaccines; this could boost neutralizing and non-neutralizing antibody responses by eliciting a more robust $\mathrm{CD} 4^{+} \mathrm{T}$ cell response and would engage a greater $\mathrm{CD} 8^{+} \mathrm{T}$ cell response that had been primed during a prior infection. Eliciting more robust antibody and $\mathrm{CD} 8^{+} \mathrm{T}$ cell responses against non-S epitopes during vaccination could be important, as there are probably
non-S targets of immunity that promote the resolution of infections and that can mediate broad immunity against SARS-CoV-2 variants.

A central question related to SARS-CoV-2 immunity is whether infection establishes a reservoir of memory cells against this pathogen that are capable of fending off a subsequent infection. This study is reassuring, as the majority of individuals who were infected six months earlier, even if they experienced no symptoms or mild symptoms during infection, were capable of mounting a cellular immune response against this pathogen. However, significant heterogeneity in the $\mathrm{T}$ cell response was observed, and thus future studies will need to define whether the early $\mathrm{T}$ cell response predicts the quality of antibodies elicited by infection and which host or viral factors other than initial disease severity predict the magnitude, quality and/or durability of SARS-CoV-2 immunity. From there, we can build an understanding of immune correlates of protection against long-term clinical outcomes such as post-acute COVID syndrome and/or reinfection, including with SARS-CoV-2 variants (Fig. 1).

Prasanna Jagannathan $n^{1,2}$ and Taia T. Wang (iD) 1,2,3凶

${ }^{1}$ Department of Medicine, Division of Infectious Diseases, Stanford University, Stanford, CA, USA. ${ }^{2}$ Department of Microbiology and Immunology, Stanford University, Stanford, CA, USA. ${ }^{3}$ Chan Zuckerberg Biohub, San Francisco, CA, USA.

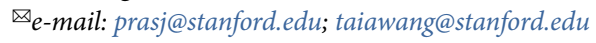

Published online: 19 April 2021

https://doi.org/10.1038/s41590-021-00923-3

References

1. Grifoni, A. et al. Cell 181, 1489-1501.e15 (2020).

2. Sekine, T. et al. Cell 183, 158-168.e14 (2020).

3. Rydyznski Moderbacher, C. et al. Cell 183, 996-1012.e19 (2020).

4. Braun, J. et al. Nature 587, 270-274 (2020).

5. Dan, J. M. et al. Science 371, eabf4063 (2021).

6. Zuo, J. et al. Nat. Immunol. https://doi.org/10.1038/s41590-02100902-8 (2021).

7. Sattler, A. et al. J. Clin. Invest. 130, 6477-6489 (2020).

8. Hansen, C. H., Michlmayr, D., Gubbels, S. M., Mølbak, K. \& Ethelberg, S. Lancet https://doi.org/10.1016/S01406736(21)00575-4 (2021).

9. Locci, M. et al. Immunity 39, 758-769 (2013).

\section{Acknowledgements}

Support was received from Stanford University, the Chan Zuckerberg Biohub and the Searle Scholars Program.

Research reported in this publication was supported by Fast Grants, CEND COVID Catalyst Fund and the National Institute of Allergy and Infectious Diseases of the National Institutes of Health under award nos. U19AI111825, U54CA260517, U01AI150741-01S1 and R01AI139119.

Competing interests

The authors declare no competing interests. 